\title{
One-Anastomosis and Roux-en-Y Gastric Bypass Promote Similar Weight Loss, Patient Satisfaction, Quality of Life, Inflammation Grade, and Cellular Damage in the Esophagus and Gastric Pouch in a Short-term Follow-up
}

\author{
Rafael C. Katayama', Carlos H. Arasaki' ${ }^{1}$, Fernando A.M. Herbella ${ }^{2}$, Ricardo A. Neto ${ }^{2}$, Gaspar de Jesus Lopes Filho ${ }^{1}$ \\ 'Department of Surgery, Division of Surgical Gastroenterology, Universidade Federal de São Paulo-UNIFESP, São Paulo; ${ }^{2}$ Department of Pathology, Escola Paulista de
} Medicina-EPM, São Paulo, Brazil

Background: One-anastomosis gastric bypass (OAGB) is a simpler procedure than Roux-en-Y gastric bypass (RYGB); however, biliary reflux can occur and impair outcomes. This study aimed to compare outcomes of OAGB and RYGB.

Methods: Twenty patients with morbid obesity were randomized prospectively into two groups: OAGB ( $n=10)$ or RYGB ( $n=10)$. Quality of life (36-item short-form health survey [SF-36]), satisfaction (Visick scale), and body mass index (BMI) were evaluated before and 6 months after the operation. All patients underwent esophagogastroduodenoscopy with gastric and esophageal mucosal biopsies at 3 and 6 months after their operation.

Results: The study found no significant difference in BMI before surgery (OAGB, $43.2 \mathrm{~kg} / \mathrm{m}^{2} ; \mathrm{RYGB}, 43.1 \mathrm{~kg} / \mathrm{m}^{2}$; $P=0.90$ ) or at 6 months postoperative (OAGB, $\left.32.1 \mathrm{~kg} / \mathrm{m}^{2} ; \mathrm{RYGB}, 31.8 \mathrm{~kg} / \mathrm{m}^{2} ; P=0.91\right)$. There was no significant difference in improvement of quality of life (four SF-36 domains) or satisfaction ( $P=0.08$ ) between groups at 6 months. There was no statistical difference between gastric $(P=0.10)$ and esophageal $(P=0.76)$ inflammation grade at three or 6 months between the two groups.

Conclusion: OAGB and RYGB are equally effective in terms of weight loss, patient satisfaction, and quality of life improvement at 6 months after the procedures. Inflammation grade and cellular damage in the gastric pouch and in the esophagus were similar.

Key words: One-anastomosis gastric bypass, Roux-en-Y gastric bypass, Biliary reflux, Morbid obesity, Quality of life, Weight loss

\author{
Received April 3, 2021 \\ Reviewed September 4, 2021 \\ Accepted September 23, 2021 \\ ${ }^{*}$ Corresponding author \\ Rafael C. Katayama \\ (iD \\ https://orcid.org/0000-0001-9403-8971 \\ Department of Surgery, Division of \\ Surgical Gastroenterology, Universidade \\ Federal de São Paulo-UNIFESP, \\ 1123 Tabapua St, rm 21, Sao Paulo, \\ SP 04533-010, Brazil \\ Tel: +55-11-31673887 \\ Fax: +55-11-31673887 \\ E-mail: caue.katayama@unifesp.br
}

\section{INTRODUCTION}

One-anastomosis gastric bypass (OAGB), described by Rutledge ${ }^{1}$ in 2001, is increasing in popularity due to its simpler procedure than Roux-en-Y gastric bypass (RYGB) with similar effectiveness and complication rate. ${ }^{2}$ Despite the enthusiasm of some re- garding early excellent OAGB results, ${ }^{1}$ others are concerned about the possibility of deleterious biliary reflux and its long-term consequences secondary to a gastrojejunal anastomosis similar to that of Billroth II anastomosis. ${ }^{2,3}$ This study aimed to compare weight loss, patient satisfaction, quality of life, and esophageal and gastric mucosal damage of OAGB and RYGB.

Copyright ( $(2021$ Korean Society for the Study of Obesity

(a) This is an Open Access article distributed under the terms of the Creative Commons Attribution Non-Commercial License (https://creativecommons.org/licenses/by-nc/4.0/) which permits unrestricted non-commercial use, distribution, and reproduction in any medium, provided the original work is properly cited. 


\section{METHODS}

\section{Patient selection}

We prospectively randomized 20 morbidly obese patients into two groups: (1) OAGB group $(\mathrm{n}=10): 10$ females, mean age 39 years and (2) RYGB group $(n=10)$ : 9 females, mean age 42 years. Inclusion criteria were consecutive candidates for bariatric operation irrespective of sex and ethnicity, age between 20-60 years, and body mass index (BMI) between $35 \mathrm{~kg} / \mathrm{m}^{2}$ and $50 \mathrm{~kg} / \mathrm{m}^{2}$. Exclusion criteria were esophagitis grade C or D or Barrett's esophagus, previous foregut operation, and refusal to provide informed consent.

\section{Randomization}

Patients were randomized to one of the two groups at the time of surgery. Patients were blinded to the procedure until the end of the study.

\section{Surgical procedures}

All procedures were performed by laparotomy by the same surgeon (RCK).

\section{OAGB procedure}

The technique utilized in this study followed Rutledge's original description. ${ }^{4}$ In summary, a $10-\mathrm{cm}$ pouch calibrated along a $32-\mathrm{Fr}$ bougie was created with the aid of staplers. An antecolic end-toside manual gastrojejunostomy was performed using a jejunal loop $200 \mathrm{~cm}$ from the ligament of Treitz with a $2-\mathrm{cm}$ diameter.

\section{RYGB procedure}

A 4-6-cm pouch calibrated along a 32-Fr bougie was created with the aid of staplers. The Roux-en-Y was performed with a $50-\mathrm{cm}$ biliopancreatic limb length and 100-cm alimentary limb length. The retrocolic/retrogastric end-to-side gastrojejunal anastomosis was performed with a 2 -cm diameter.

\section{Clinical evaluation}

Patients underwent a routine work-up for bariatric operations. BMI was measured before the operation and at 6 months after the procedure. Patient satisfaction was evaluated at 6 months after the operation with the aid of the modified Visick Scale. ${ }^{5}$ Quality of life was evaluated before and at 6 months after the operation with the aid of the medical outcomes study 36 -item short-form health survey (SF-36), validated for local language. ${ }^{6}$ The SF-36 questionnaire is divided into domains. Each domain is scored from 0 to 100 , with a higher score indicating better patient performance in the assessed domain.

\section{Endoscopic evaluation}

All patients underwent an esophagogastroduodenoscopy (EGD) before and at 3 and 6 months after the operation. Esophageal and gastric biopsies were performed for histopathological evaluation. Presence of Helicobacter pylori (HP) was investigated by histopathological evaluation, and positive patients were treated before the operation. The presence of bile in the stomach or esophagus was recorded.

\section{Histopathological evaluation}

The endoscopic biopsy specimens were fixed and stained with hematoxylin-eosin, and the reading was performed by a single pathologist. Classification was performed using the modified Sydney classification system (Houston-Updated Sidney System). ${ }^{7}$ According to this system, the degree of acute inflammation/activity is assessed through the numbers of polymorphonuclear cells and leukocytes in the lamina propria. Chronic inflammation is assessed through the numbers of lymphocytes and histiocytes in the lamina propria according to a visual scale. We also evaluated the presence or absence of cystic glandular degeneration. This cell degeneration commonly is related to reactive gastropathy secondary to bile reflux. The same system was adapted and applied for esophagitis inflammation grading.

\section{Statistics}

Data are expressed as mean \pm standard deviation, median (range), or frequency (\%). Student t-test was used to compare age and the Mann-Whitney test to compare comorbidities. Multivariate analysis of BMI and SF-36 domains between time and groups was calculated with the aid of analyses of variance. The generalized estimating equation was chosen to compare the presence of $\mathrm{HP}$, gastritis, and esophagitis grade among times and between groups. A $P<0.05$ was considered significant. IBM SPSS version 23.0 (IBM Corp., 
Armonk, NY, USA) was used to assist in the analysis.

\section{Ethics}

The protocol was approved by the local ethics committee (No. CAAE 65970217.3.000.5505), and all patients provided informed consent.

\section{RESULTS}

Groups were similar in age, sex, ethnicity, comorbidities, and preoperative esophagitis grade (Table 1). There was no significant difference in BMI for the groups either preoperatively or at 6 months; however, a significant reduction in BMI was seen at 6 months in both groups (Table 2).

There was no significant difference in Visick scale between groups at the 6-month follow-up (Table 1) or in any SF-36 domain between groups before the operation. At the 6-month follow-up, regardless of group, there was improvement in vital capacity and general health and vitality scores, but not in physical performance, pain, social, emotional, or mental health scores. There was no significant difference in scores at the 6-month follow-up between groups (Table 2).

There was no significant difference in HP infection between groups (Table 3 ) or in esophagitis grade between groups before or after the operation. Bile was not recorded in any EGD. We found no significant difference in acute or chronic gastric inflammation grade before or after the operation. Glandular cystic degeneration was significantly lower preoperatively than at 3 and 6 months, but there was no difference in the comparison between group $\times$ time.

\section{DISCUSSION}

In 2001, Rutledge proposed a new technique named OAGB, which has gained many followers in recent years as studies have shown similar effectiveness in sustained weight loss and comorbidity control to RYGB and similar complication rates. ${ }^{2,3}$ OAGB is simpler and has a shorter operative time than does RYGB. However, a primary concern is related to biliary reflux inherent to Billroth II anastomosis.

In the past, Billroth II anastomosis was widely used after gastrec- tomy for duodenal and gastric ulcer complications or gastric cancer. ${ }^{8}$ Most studies have shown that there was significant biliary reflux associated with those procedures. ${ }^{9}$ After the development of proton pump inhibitors, those operations became rare. Moreover, Roux-en-Y anastomosis started to gain the preference of surgeons to prevent biliary reflux. ${ }^{9}$ Several studies have shown higher grades of gastric remnant inflammation after Billroth II anastomosis compared to Roux-en-Y anastomosis, ${ }^{9,10}$ which could culminate in remnant gastric cancer. ${ }^{11}$ Another concern is whether biliary reflux could reach the esophagus and promote esophageal inflammation culminating in a Barrett's esophagus. ${ }^{12,13}$ However, OAGB is a dif-

Table 1. Demographic characteristics, comorbidities, operative time, preoperative upper digestive endoscopy, and Visick scale of OAGB and RYGB groups

\begin{tabular}{|c|c|c|c|c|}
\hline Variable & OAGB $(n=10)$ & $\operatorname{RYGB}(n=10)$ & Total $(n=20)$ & $P$ \\
\hline Age (yr) & & & & 0.47 \\
\hline Mean \pm SD & $39.5 \pm 7.0$ & $42.2 \pm 9.3$ & $40.9 \pm 8.1$ & \\
\hline Median (range) & $39.0(28-49)$ & $38.5(31-57)$ & $38.5(28-57)$ & \\
\hline Ethnicity & & & & 0.86 \\
\hline White & $5(50)$ & $6(60)$ & $11(55)$ & \\
\hline Black & $2(20)$ & $2(20)$ & $4(20)$ & \\
\hline Brown & $3(30)$ & $2(20)$ & $5(25)$ & \\
\hline Number of comorbidities & & & & 0.63 \\
\hline Mean \pm SD & $1.3 \pm 1.4$ & $1.5 \pm 1.2$ & $1.4 \pm 1.3$ & \\
\hline Median (range) & $1.0(0-4)$ & $2.0(0-3)$ & $1.5(0-4)$ & \\
\hline Arterial hypertension & & & & 0.36 \\
\hline No & $6(60)$ & $3(30)$ & $9(45)$ & \\
\hline Yes & $4(40)$ & $7(70)$ & $11(55)$ & \\
\hline T2DM & & & & 0.14 \\
\hline No & $9(90)$ & $5(50)$ & $14(70)$ & \\
\hline Yes & $1(10)$ & $5(50)$ & $6(30)$ & \\
\hline Other comorbidities & & & & 0.64 \\
\hline No & $5(50)$ & $7(70)$ & $12(60)$ & \\
\hline Yes & $5(50)$ & $3(30)$ & $8(40)$ & \\
\hline Operative time (min) & & & & $<0.01$ \\
\hline Mean \pm SD & $78.0 \pm 13.4$ & $125.5 \pm 10.9$ & $101.8 \pm 27.1$ & \\
\hline Median (range) & 70.0 (65-100) & $125.0(105-145)$ & $102.5(65-145)$ & \\
\hline Preoperative UDE & & & & 1.00 \\
\hline With esophagitis & $3(30)$ & $3(30)$ & $6(30)$ & \\
\hline Without esophagitis & $7(70)$ & $7(70)$ & $14(70)$ & \\
\hline Post-6-month Visick scale & & & & 0.08 \\
\hline 1 & $8(80)$ & $5(50)$ & $13(65)$ & \\
\hline$\|$ & 0 & $3(30)$ & $3(15)$ & \\
\hline III & $2(20)$ & $2(20)$ & $4(20)$ & \\
\hline IV & 0 & 0 & 0 & \\
\hline
\end{tabular}

Values are presented as number (\%) unless otherwise indicated. OAGB, one-anastomosis gastric bypass; RYGB, Roux-en-Y gastric bypass; SD, standard deviation; T2DM, type 2 diabetes mellitus; UDE, upper digestive endoscopy. 
ferent technique from those performed before. The gastric pouch is long and narrow with a straight anastomosis, which could avoid reflux, mainly to the esophagus. Moreover, a 200-cm limb length from the Treitz could decrease biliary reflux since bile can be partially absorbed and metabolized along the path between the duodenum and the stomach.

\section{Weight loss after OAGB or RYGB}

The nadir weight usually is achieved within 12 months after OAGB, with approximately $70 \%-80 \%$ of the excess weight loss is achieved at 6 months. ${ }^{3}$ A similar weight loss of the procedures was noted in a short-term follow-up (6 months). This result was expected as the anatomical variances of the techniques does not seem to affect weight loss. The size of the gastric pouch does not correlate to weight loss. ${ }^{14}$ Also, some studies have demonstrated no difference in weight loss outcomes when different RYGB limb lengths are compared, especially when the variation is between 1 and 2 meters. ${ }^{15}$

\section{Satisfaction and quality of life after OAGB or RYGB}

Satisfaction and quality of life with the OAGB could be impaired by insufficient weight loss or onset of new symptoms linked to biliary reflux. Our study showed similar high grades of satisfaction and quality of life improvement after the procedures in concordance with similar weight loss and postoperative symptoms between the procedures.

\section{Biliary reflux after OAGB or RYGB}

Previous studies are controversial as to the incidence and conse-

Table 2. Comparison of SF-36 domain and BMl at before and after 6 months from surgery between OAGB and RYGB

\begin{tabular}{|c|c|c|c|c|c|c|}
\hline \multirow{2}{*}{ SF-36 domain } & \multicolumn{2}{|c|}{ Preoperative } & \multicolumn{2}{|c|}{6 Months postoperative } & \multicolumn{2}{|c|}{$P$} \\
\hline & $\operatorname{OAGB}(n=10)$ & $\operatorname{RYGB}(n=10)$ & $\operatorname{OAGB}(n=10)$ & $\operatorname{RYGB}(n=10)$ & OAGB $\times R Y G B$ & Pre $\times$ post \\
\hline Functional capacity & & & & & 0.90 & $<0.01$ \\
\hline Median (range) & $45.0(15-95)$ & $45.0(0-80)$ & $90.0(50-100)$ & $87.5(70-100)$ & & \\
\hline Physical performance & & & & & 0.91 & 0.07 \\
\hline Mean $\pm S D$ & $56.8 \pm 40.5$ & $50.0 \pm 37.3$ & $75.0 \pm 29.6$ & $69.0 \pm 35.7$ & & \\
\hline Pain & & & & & 0.30 & 0.17 \\
\hline Mean $\pm S D$ & $64.9 \pm 34.1$ & $54.1 \pm 16.6$ & $65.6 \pm 28.3$ & $63.7 \pm 16.9$ & & \\
\hline Median (range) & $64.0(0-100)$ & $51.0(41-100)$ & $64.0(22-100)$ & $62.0(41-100)$ & & \\
\hline General health & & & & & 0.56 & $<0.01$ \\
\hline Mean \pm SD & $53.0 \pm 14.8$ & $45.2 \pm 22.0$ & $76.3 \pm 10.3$ & $75.7 \pm 17.9$ & & \\
\hline Median (range) & $55.0(5-100)$ & $54.0(20-75)$ & $90.0(30-100)$ & 75.0 (55-90) & & \\
\hline Social aspect & & & & & 0.77 & 0.62 \\
\hline Mean \pm SD & $70.5 \pm 35.5$ & $76.4 \pm 29.1$ & $75.0 \pm 30.1$ & $77.8 \pm 26.9$ & & \\
\hline Median (range) & $75.0(0-100)$ & $88.1(25-100)$ & $87.5(38-100)$ & $88.8(25-100)$ & & \\
\hline Emotional aspect & & & & & 0.76 & 0.53 \\
\hline Mean \pm SD & $66.7 \pm 42.2$ & $76.7 \pm 31.6$ & $72.7 \pm 38.9$ & $86.7 \pm 32.2$ & & \\
\hline Median (range) & $100.0(0-100)$ & 100.0 (33-100) & $100.0(0-100)$ & $100.0(0-100)$ & & \\
\hline Mental Health & & & & & 0.22 & 0.94 \\
\hline Mean $\pm S D$ & $72.0 \pm 24.7$ & $56.4 \pm 11.5$ & $77.1 \pm 19.8$ & $70.4 \pm 19.2$ & & \\
\hline Median (range) & $80.0(16-96)$ & $56.0(40-72)$ & $88.0(48-96)$ & $66.0(48-96)$ & & \\
\hline
\end{tabular}

SF-36, 36-item short-form survey; BMI, body mass index; OAGB, one-anastomosis gastric bypass; RYGB, Roux-en-Y gastric bypass; SD, standard deviation. 
quence of biliary reflux after OAGB. Perhaps, this is related to the fact that most studies are retrospective and with questionable methodology to evaluate duodenogastroesophageal reflux. Some of the studies applied symptoms questionnaires only, ${ }^{2-4}$ while others employed EGD to search for bile in the esophagus and in the gastrojejunal anastomosis or to grade esophagitis. ${ }^{2,3,16}$ We combined three methods to strengthen the attempt to identify biliary reflux. Apart from similar satisfaction and quality of life improvement, no bile was reported in the esophagus or around the gastrojejunal anastomosis, and there was no difference in the inflammation grade of the stomach and esophagus at 3 or 6 months after both procedures (Table 3).

An interesting finding was increased glandular cystic degeneration in both groups at 3 and 6 months postoperative, shown in $60 \%$ of OAGB and $70 \%$ of RYGB patients (Table 3, Fig. 1). Glandular cystic degeneration commonly is described in cases of reactive gas-

Table 3. Histopathological examination of gastric and esophageal mucosal biopsies preoperatively and at 3 and 6 months after surgeries

\begin{tabular}{|c|c|c|c|c|c|c|c|c|}
\hline \multirow{2}{*}{ Parameter } & \multicolumn{2}{|c|}{ Preoperative } & \multicolumn{2}{|c|}{3 Months postoperative } & \multicolumn{2}{|c|}{6 Months postoperative } & \multicolumn{2}{|c|}{$P$} \\
\hline & $\operatorname{OAGB}(n=10)$ & $\operatorname{RYGB}(n=10)$ & OAGB $(n=10)$ & $\operatorname{RYGB}(n=10)$ & $\operatorname{OAGB}(n=10)$ & $\operatorname{RYGB}(n=10)$ & $O A G B \times R Y G B$ & Pre $\times$ post \\
\hline Gastric glandular cystic degeneration & & & & & & & 0.43 & 0.04 \\
\hline Yes & 0 & $2(20)$ & $6(60)$ & $5(50)$ & $6(60)$ & $7(70)$ & & \\
\hline No & $10(100)$ & $8(80)$ & $4(40)$ & $5(50)$ & $4(40)$ & $3(30)$ & & \\
\hline Helicobacter pylori & & & & & & & 0.21 & 0.83 \\
\hline No & $5(50)$ & $9(90)$ & $8(80)$ & $8(80)$ & $8(80)$ & $7(70)$ & & \\
\hline Yes & $5(50)$ & $1(10)$ & $2(20)$ & $2(20)$ & $2(20)$ & $3(30)$ & & \\
\hline Gastric acute and chronic inflammation grade & & & & & & & 0.10 & 0.63 \\
\hline Mean \pm SD & $3.4 \pm 2.6$ & $1.7 \pm 2.4$ & $1.7 \pm 2.2$ & $2.0 \pm 2.0$ & $1.8 \pm 2.1$ & $2.1 \pm 1.9$ & & \\
\hline Median (range) & $3.5(0-6)$ & $1.0(0-6)$ & $1.0(0-6)$ & $1.5(0-6)$ & $1.0(0-6)$ & $1.0(0-6)$ & & \\
\hline Esophagitis grade & & & & & & & 0.77 & 0.42 \\
\hline 0 & NA & NA & $4(40)$ & $2(20)$ & $3(30)$ & $1(10)$ & & \\
\hline 1 & NA & NA & $4(40)$ & $5(50)$ & $3(30)$ & $6(60)$ & & \\
\hline 2 & NA & NA & $1(10)$ & $3(30)$ & $4(40)$ & $2(20)$ & & \\
\hline 3 & NA & NA & $1(10)$ & 0 & 0 & $1(10)$ & & \\
\hline Bile on EGD & 0 & 0 & 0 & 0 & 0 & 0 & & \\
\hline
\end{tabular}

Values are present as number (\%) unless otherwise indicated.

OAGB, one-anastomosis gastric bypass; RYGB, Roux-en-Y gastric bypass; SD, standard deviation; NA, not available; EGD, esophagogastroduodenoscopy.
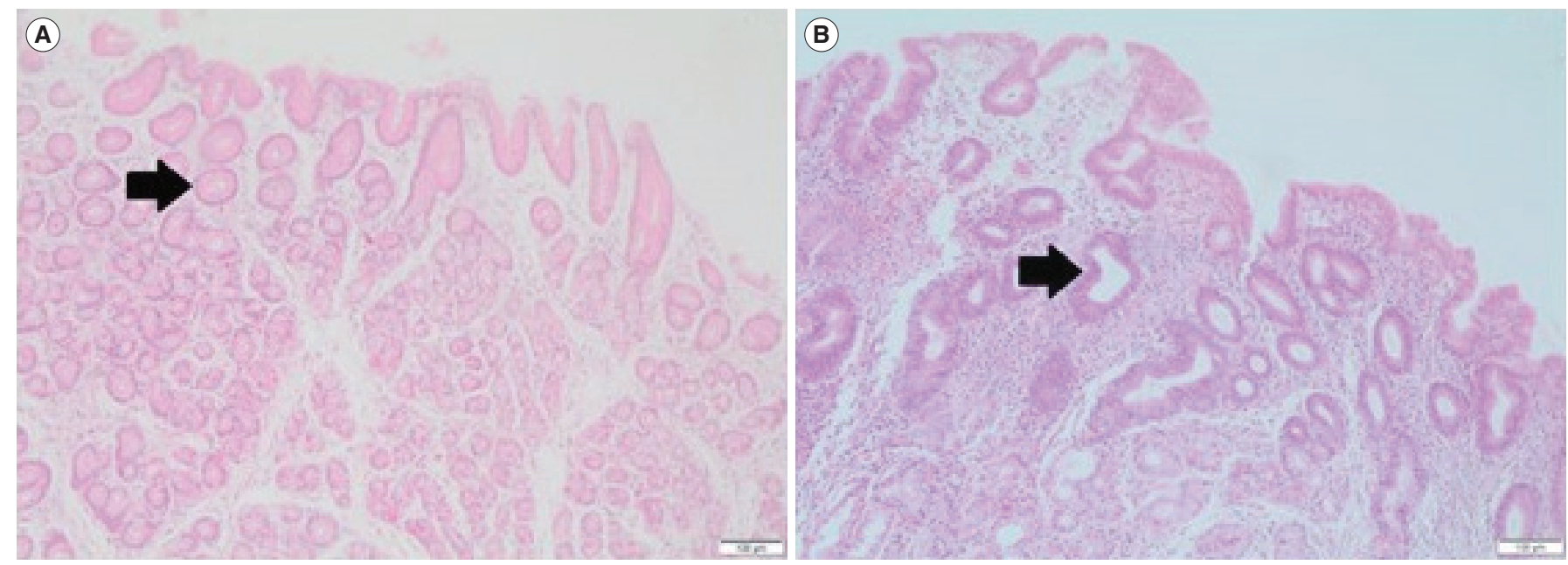

Figure 1. Endoscopic biopsy specimens fixed and stained with hematoxylin \& eosin. Magnification, x100. (A) Microscopy photo of normal gastric mucosa cell (arrow). (B) Microscopy photo of glandular cystic degeneration (arrow). 
tropathy, often related to biliary reflux. ${ }^{17}$ Nevertheless, we believe that cellular damage is inherent to gastroplasty with gastro-jejunal anastomosis regardless of reconstruction type or secondary to impaction of food promoted by a narrow anastomosis. Therefore, it was not possible to associate the glandular cystic degeneration with the presence of biliary reflux since we found similar percentages of cellular damage in the two groups.

In conclusion, OAGB was shown to be a simpler operation with similar outcomes for weight loss, satisfaction, and quality of life compared to RYGB. Biliary reflux is the major concern for OAGB; however, we could not find different degrees of gastric or esophageal inflammation associated with this technique compared to RYGB. Therefore, OAGB seems to be a promising procedure.

\section{Limitations and strengths}

The study sample was small; however, it was a prospective randomized trial combining three methods (endoscopic, histopathological, and clinical evaluations) for the analysis of biliary reflux. Operations were performed by laparotomy since the participating institution did not have resources for laparoscopic surgery; however, we believe the route does did not affect the results.

\section{CONFLICTS OF INTEREST}

The authors declare no conflict of interest.

\section{ACKNOWLEDGMENTS}

We thank Dr. Iuri Tamasauskas, Dr. Marcelo Aniche, and Eldir Martinelli for being part of the surgical team and Dr. Heitor Côrrea Barbin for the esophagogastro-duodenoscopy.

\section{AUTHOR CONTRIBUTIONS}

Study concept and design: RCK and CHA; acquisition of data: RCK; analysis and interpretation of data: RAN, RCK, CHA, and FAMH; drafting of the manuscript: RCK, CHA, and FAMH; critical revision of the manuscript: RCK; statistical analysis: RCK, $\mathrm{CHA}$, and FAMH; administrative, technical, or material support: GJLF; and study supervision: GJLF.

\section{REFERENCES}

1. Rutledge R. The mini-gastric bypass: experience with the first 1,274 cases. Obes Surg 2001;11:276-80.

2. Robert M, Espalieu P, Pelascini E, Caiazzo R, Sterkers A, Khamphommala L, et al. Efficacy and safety of one anastomosis gastric bypass versus Roux-en-Y gastric bypass for obesity (YOMEGA): a multicentre, randomised, open-label, non-inferiority trial. Lancet 2019;393:1299-309.

3. Parmar CD, Mahawar KK. One anastomosis (mini) gastric bypass is now an established bariatric procedure: a systematic review of 12,807 patients. Obes Surg 2018;28:2956-67.

4. Rutledge R, Kular K, Manchanda N. The mini-gastric bypass original technique. Int J Surg 2019;61:38-41.

5. Visick AH. A study of the failures after gastrectomy. Ann R Coll Surg Engl 1948;3:266-84.

6. Ciconelli RM. Validated Portuguese translation of outcomes study 36- item short -form health survey [dissertation]. São Paulo: Universidade Federal de São Paulo, Escola Paulista de Medicina;1997.

7. Rugge M, Genta RM. Staging and grading of chronic gastritis. Hum Pathol 2005;36:228-33.

8. Cooperman AM. Postoperative alkaline reflux gastritis. Surg Clin North Am 1976;56:1445-59.

9. Kim MS, Kwon Y, Park EP, An L, Park H, Park S. Revisiting laparoscopic reconstruction for billroth 1 versus billroth 2 versus Roux-en-Y after distal gastrectomy: a systematic review and meta-analysis in the modern era. World J Surg 2019;43: 1581-93.

10. Csendes A, Burgos AM, Smok G, Burdiles P, Braghetto I, Díaz JC. Latest results (12-21 years) of a prospective randomized study comparing Billroth II and Roux-en-Y anastomosis after a partial gastrectomy plus vagotomy in patients with duodenal ulcers. Ann Surg 2009;249:189-94.

11. Diogo Filho A, Botelho LF, Nishiyama A, Zumpano LE, Monte RC, Rosa SC. Gastric stump cancer after gastrectomy by gastroduodenal peptic ulcer. Arq Bras Cir Dig 2016;29:65.

12. Herbella FA, Patti MG. Gastroesophageal reflux disease: from pathophysiology to treatment. World J Gastroenterol 2010; $16: 3745-9$. 
13. Herbella FA, Schlottmann F, Patti MG. Antireflux surgery and barrett's esophagus: myth or reality? World J Surg 2018; 42:1798-802.

14. Topart P, Becouarn G, Ritz P. Pouch size after gastric bypass does not correlate with weight loss outcome. Obes Surg 2011; 21:1350-4.

15. Feng JJ, Gagner M, Pomp A, Korgaonkar NM, Jacob BP, Chu CA, et al. Effect of standard vs extended Roux limb length on weight loss outcomes after laparoscopic Roux-en-Y gastric bypass. Surg Endosc 2003;17:1055-60.
16. Saarinen T, Pietiläinen KH, Loimaala A, Ihalainen T, Sammalkorpi $\mathrm{H}$, Penttilä $\mathrm{A}$, et al. Bile reflux is a common finding in the gastric pouch after one anastomosis gastric bypass. Obes Surg 2020;30:875-81.

17. El-Zimaity H, Riddell RH. Inflammatory disorders of the stomach. In: Shepherd NA, Warren BF, Williams GT, Greenson JK, Lauwers GY, Novelli MR, editors. Morson and Dawson's gastrointestinal pathology. 5th ed. Oxford: Wiley-Blackwell; 2013. p. 110-48. 